\title{
LIFE LONG LEARNING PROCESS TO REFORM THE THEORIES AND PRACTICES OF EDUCATION SECTOR IN THE COUNTRY
}

This work was supported by the research project №21T-5C155

Poghosyan G.S., Gasparyan A.M., Amiryan E.V. (National Academy of Sciences of the Republic of Armenia, Yerevan, Armenia) gayane_surenovna@yahoo.com,anahit-movsesi@mail.ru, amiryan.1996@inbox.ru 乙tinцшјшgर्üध uर्u. 23.07.2021 9pmpunuर्uाu uर्रu. 14.08.2021

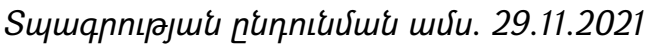

This article analyzes the theoretical foundations of lifelong learning as opportunities for a person to live and flourish in a democratic society. Continuous learning, according to the existing concept, implies that a person needs to constantly keep learning, update the acquired knowledge, skills and abilities. It should be implemented throughout life not only by means of natural communication, life experience, but also through the realization of variety conscious educational opportunities via formal, nonform and informal education. In the article, determinations, standards and objectives of the lifelong learning listed in local and international literature are stated. Detailed analysis of the authors' work, models and reports, as well as the approaches of different specialists of our country are set out.Video-methodological analysis shows that almost all European countries, including China, are shifting from adult to continuing education, and then to lifelong learning.

Keywords: lifelong education, lifelong learning, continuing education, formal, non-form and informal education.

DOI: https://doi.org/10.46991/SBMP/2022.5.1.099

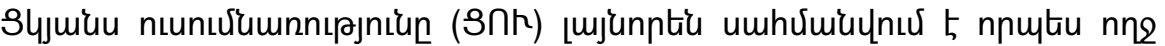

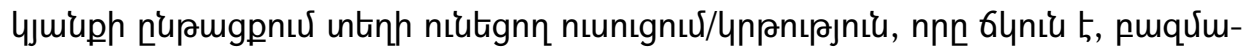

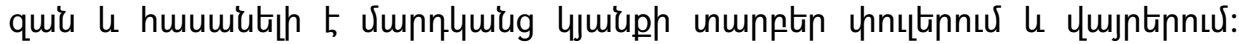

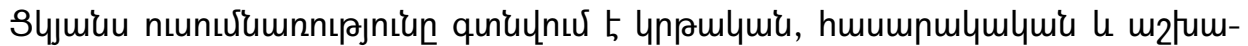

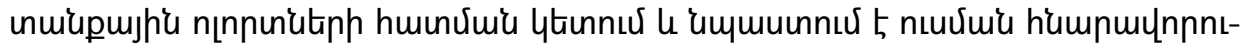

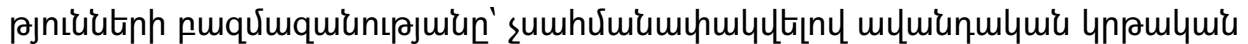

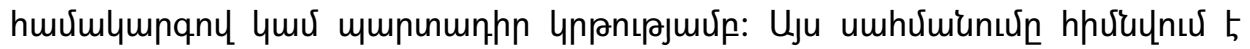

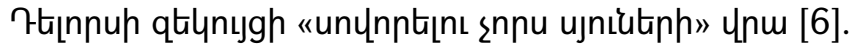

Unynnnuर tuर pưuium. 


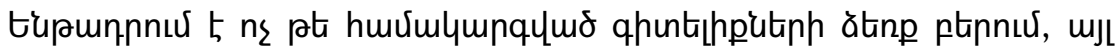

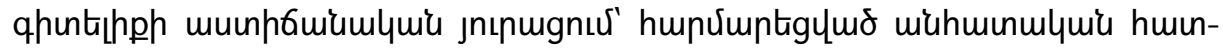
umunzititiphu, Ununnnuर्u tư mint.

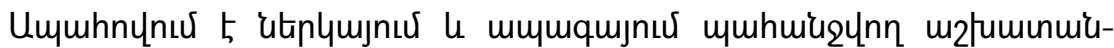

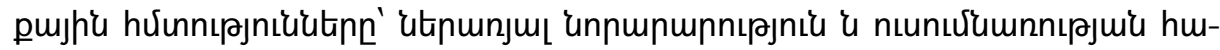

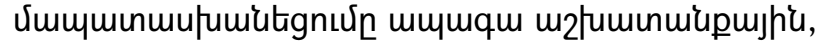
Ununnnuर्u tư qnjulygtı nıph2utiph htion.

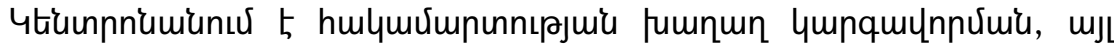

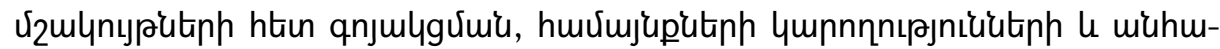

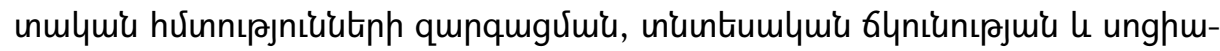

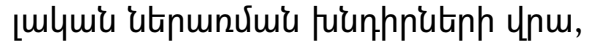

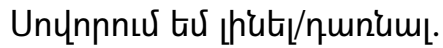

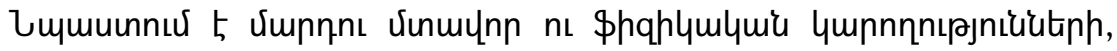

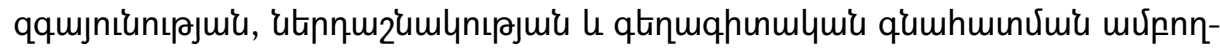
¿miluiu qunquguruin:

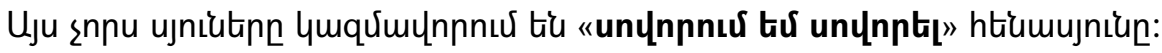

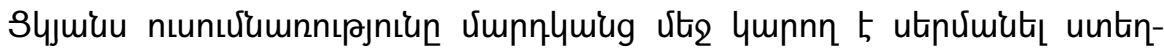

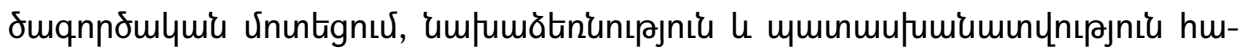

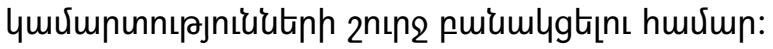

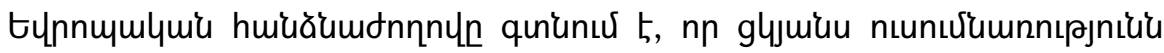

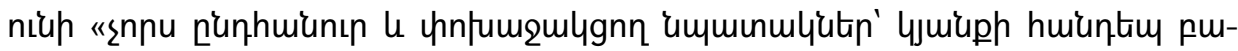

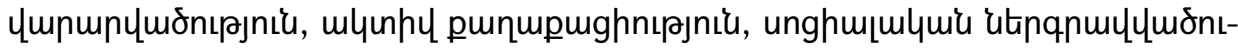

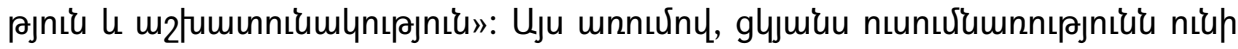

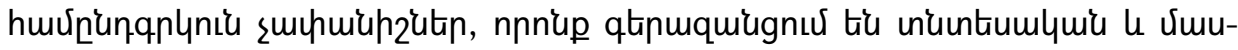
umqhunmlymi utin nınnunutinn:

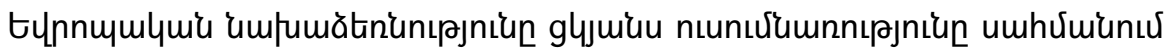

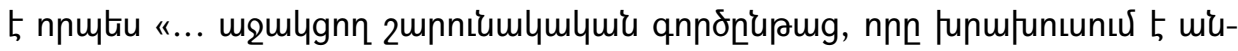

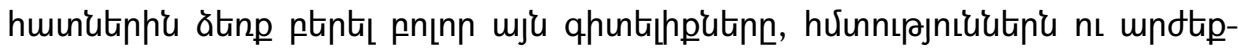

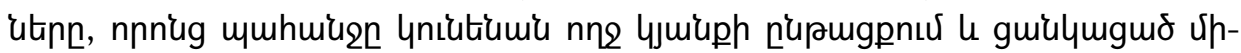

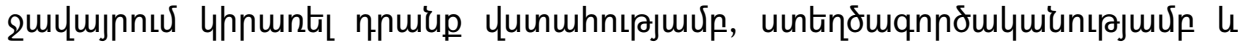
hu6nujpnu" [9]:

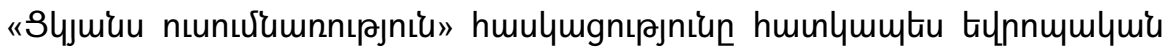

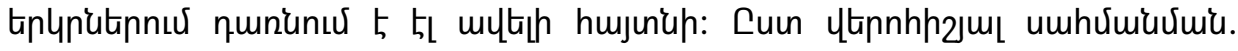

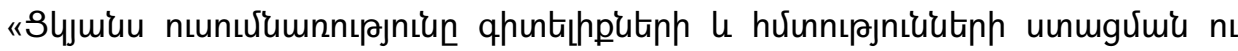

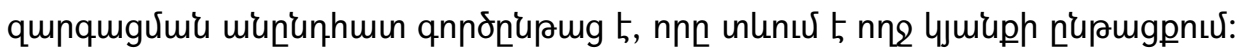

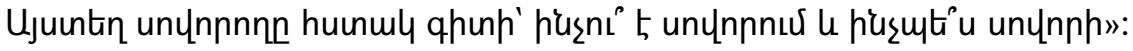

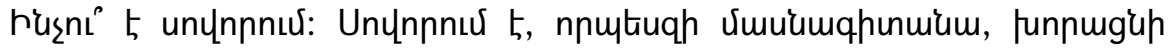

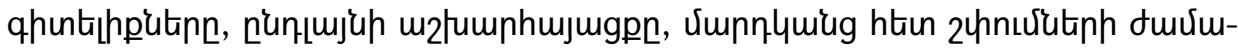




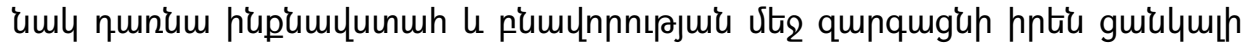
humlyuuhzitit:

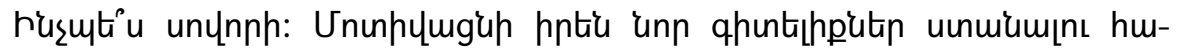

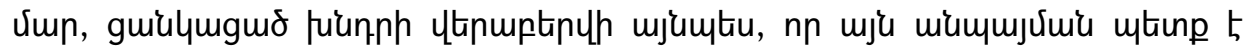

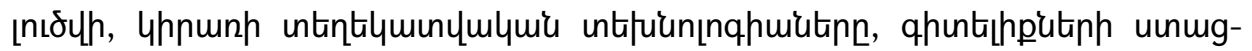

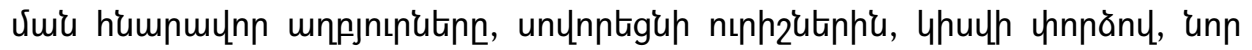

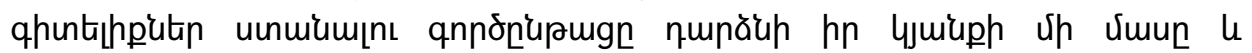
umunqumutu hu6nıp uinwium npwiuhg:

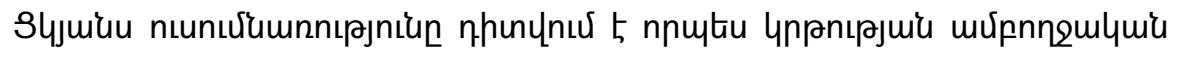

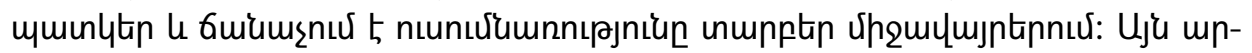

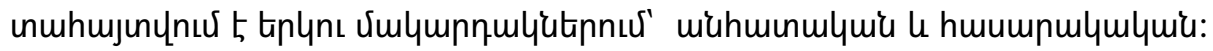

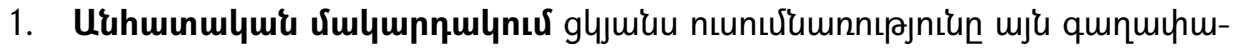

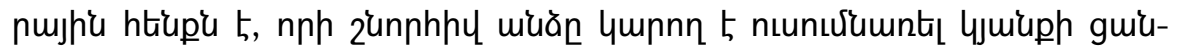

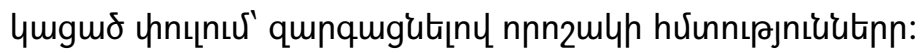

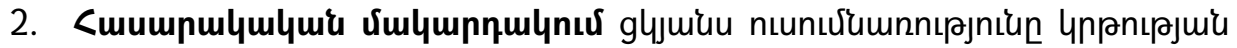

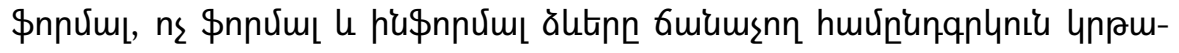
yuiu hurululump t:

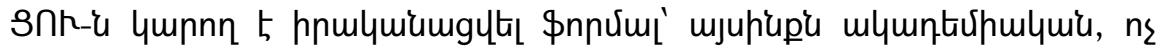

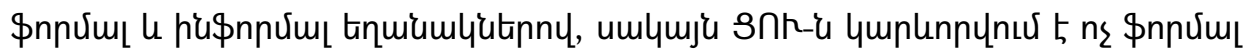

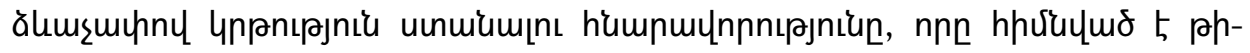

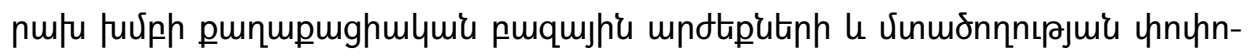

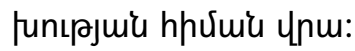

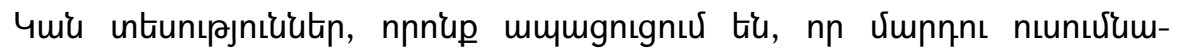

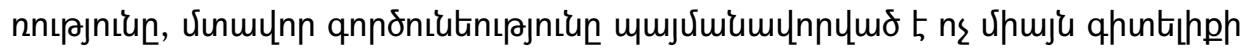

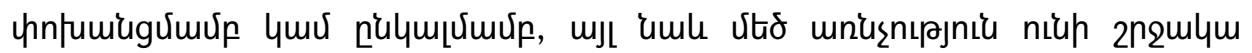

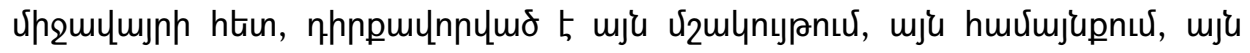

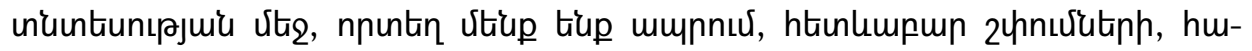

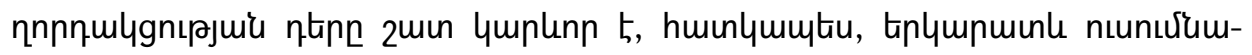

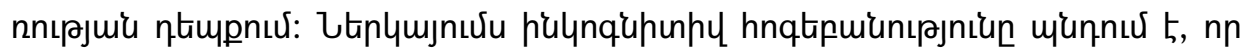

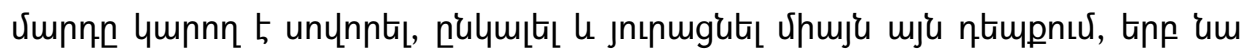

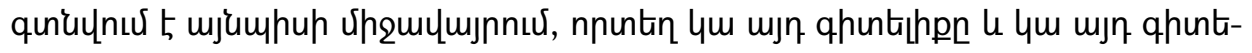

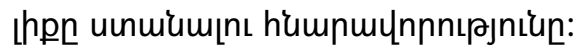

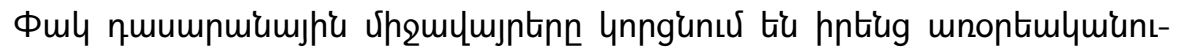

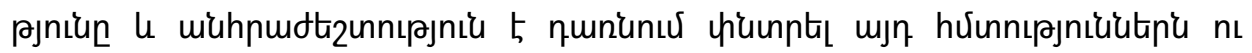

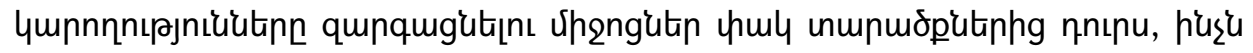

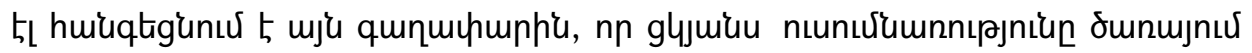

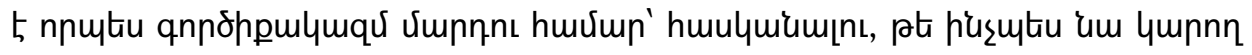

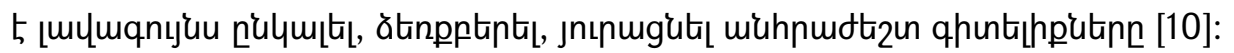

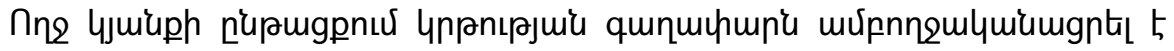

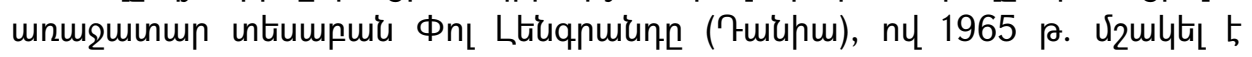




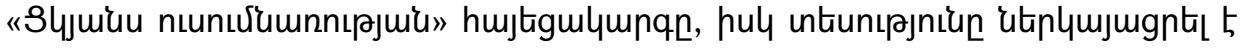

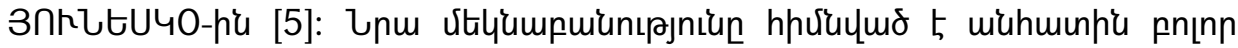

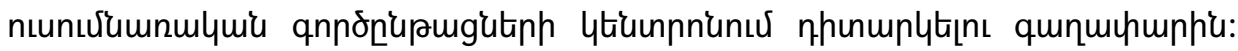

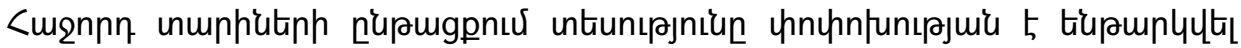

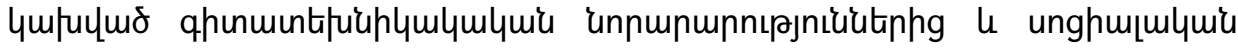
uquhuiugutinht:

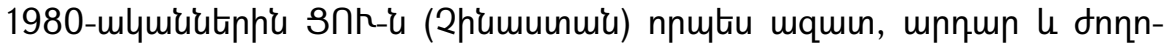

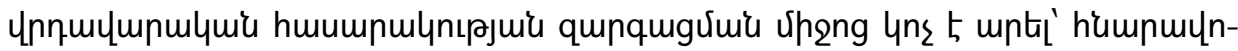

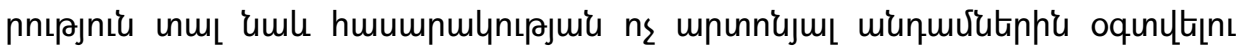

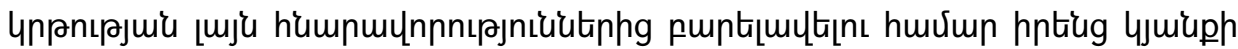
nnwly [7]:

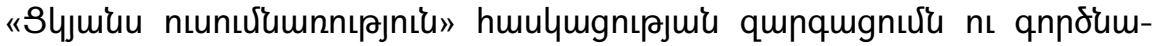

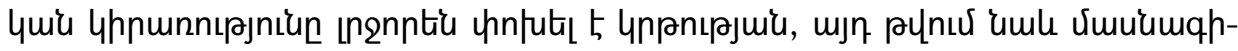

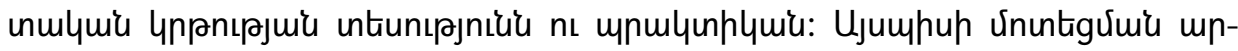

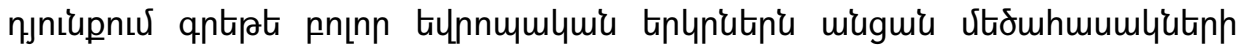

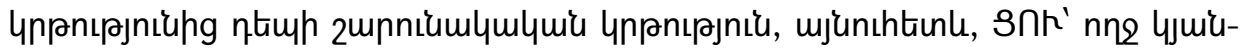

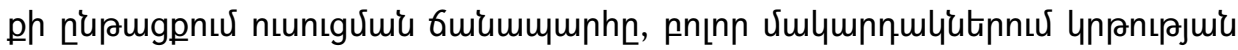

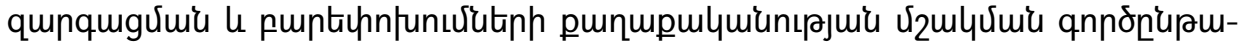

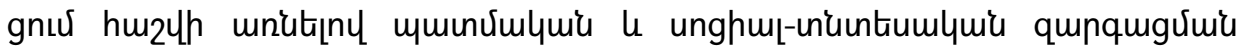

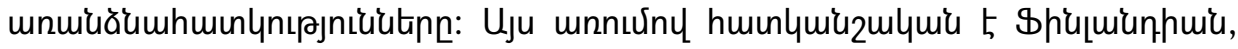

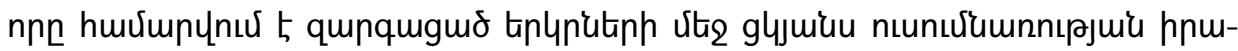

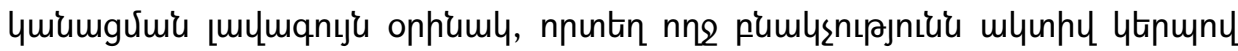

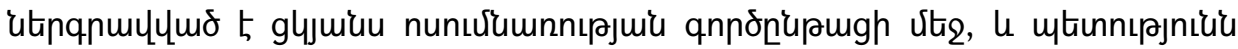
uर्utu 4tinu mquilgnuर 5 [4]:

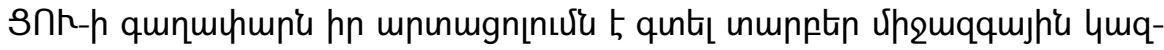

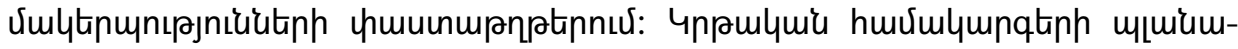

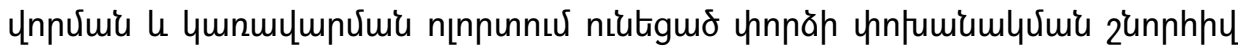

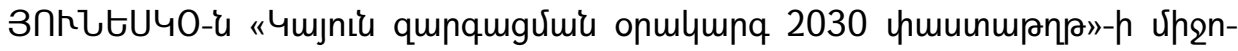

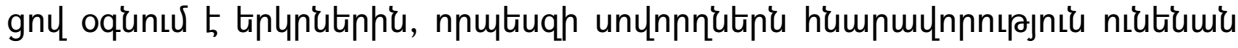

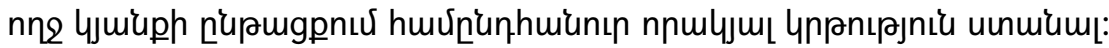

Utip unmpmóm2n

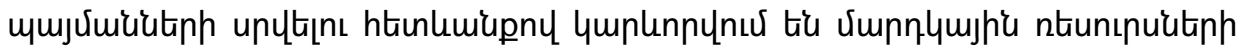

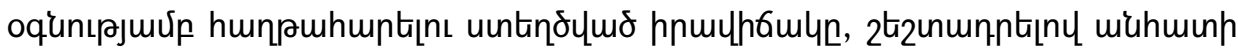

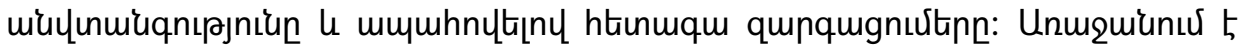

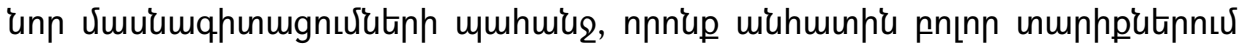

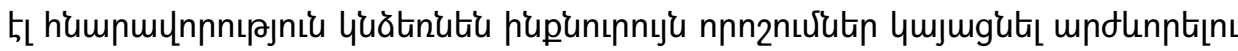

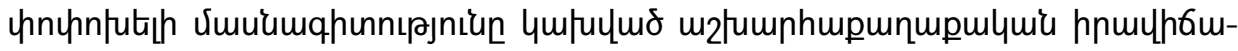

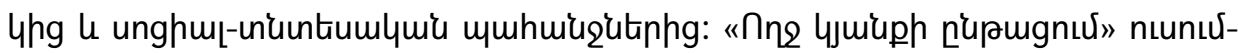

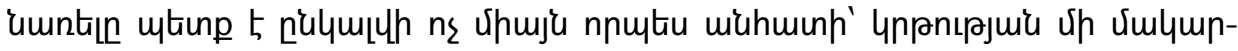

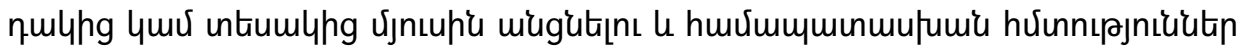




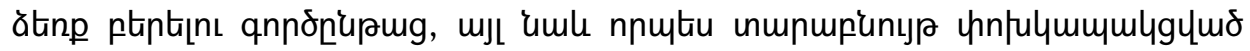

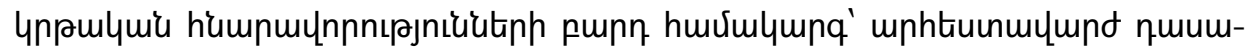

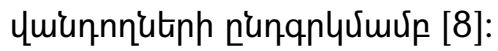

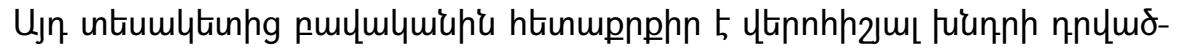

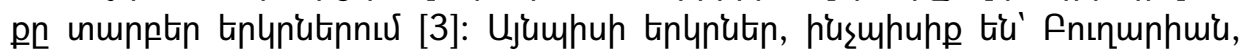

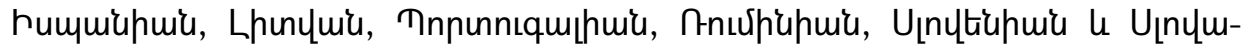

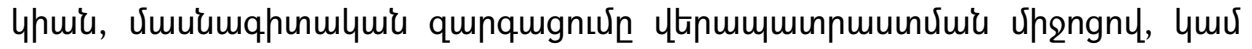

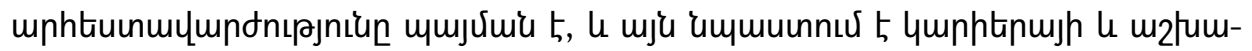

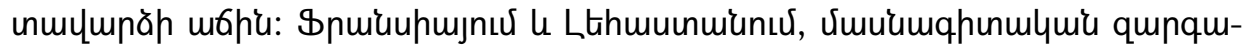

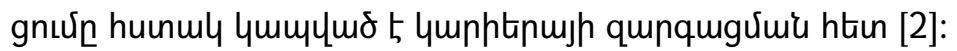

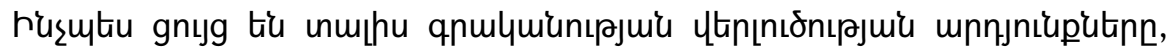

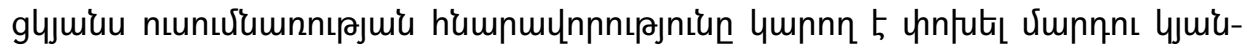

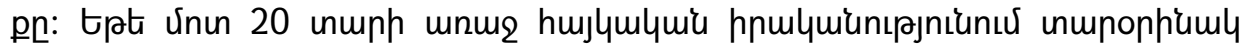

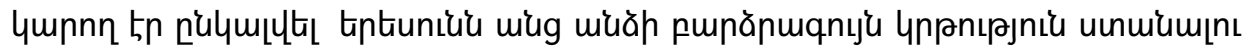

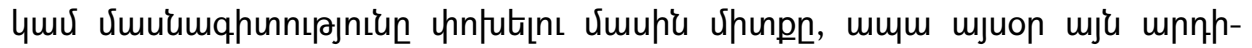
mlymint 5 :

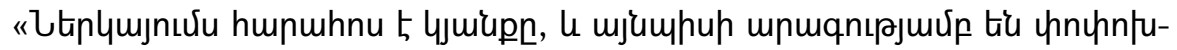

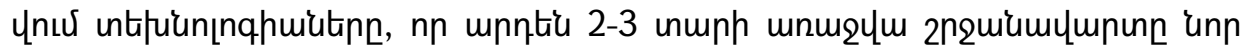

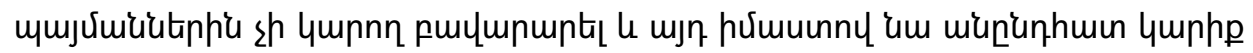

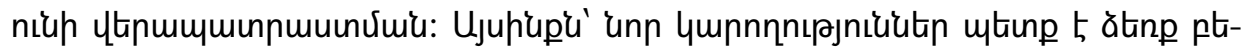

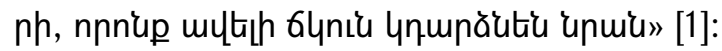

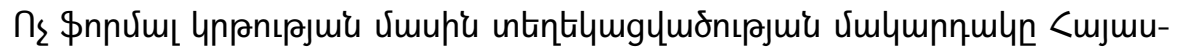

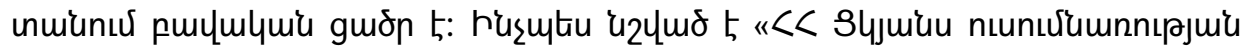

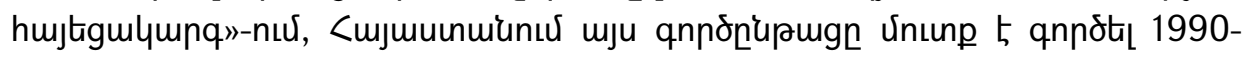

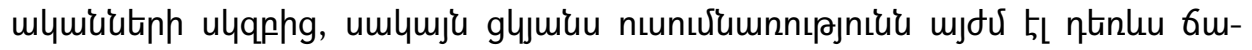

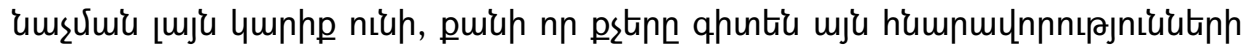

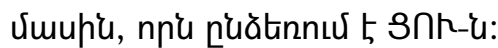

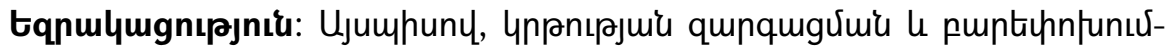

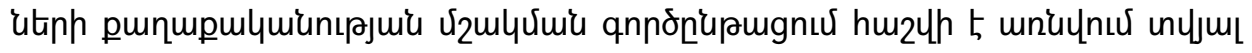

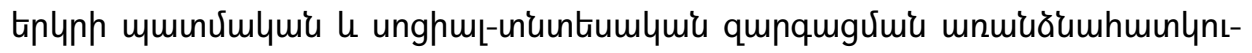
pjnicuitinn:

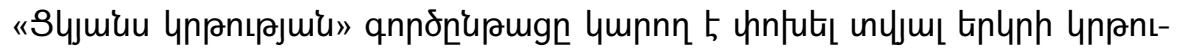

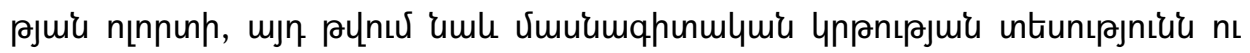
unumunhlywiu:

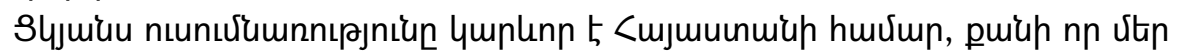

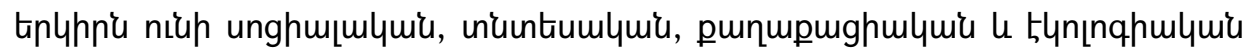

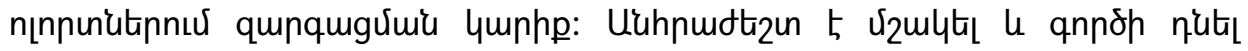

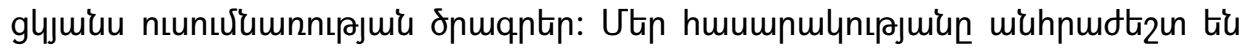

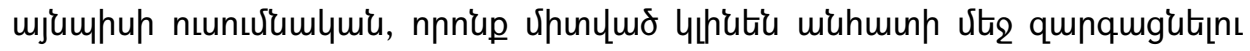

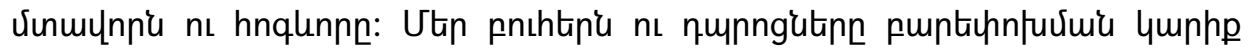




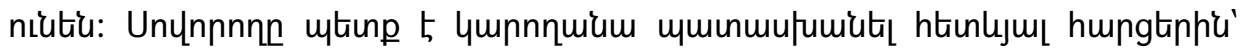

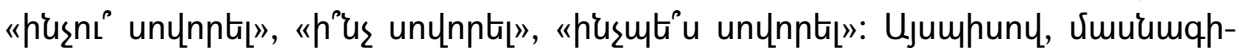

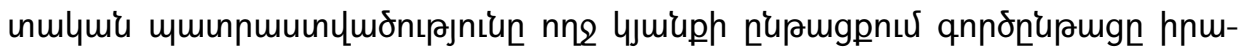

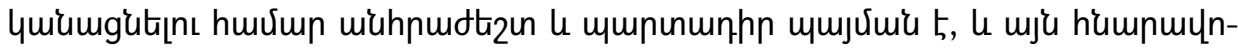

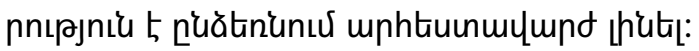

\section{9pulquínıpjnitu}

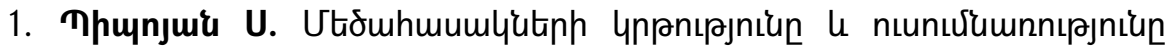

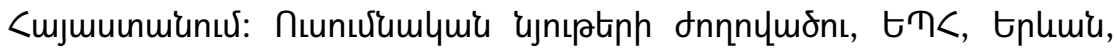
2018, 52 95-110:

2. Куля Э., Пэнковска М. Профессиональное развитие учителей в странах европейского союзакак элемент непрерывного образования, 2016, с. 54-62.

3. Погосян Г.С., Гаспарян А.М., Григорян М.М. Анализ процесса обучения на протяжении всей жизни. Молодежь и будущее: профрессиональная и личностная самореализация. Материалы $\mathrm{X}$ междун. научно-практической конференции. г. Владимир, 2021, с. 57-62.

4. Finnish National Board of Education (2014; 2015): The National Core Curriculum for Basic Education. http://www.oph.fi/English.

5. Lengrand Paul. An Introduction to Lifelong Education, UNESCO, Paris, 1971 ,

p.

99.

http://www.tandfonline.com/doi/abs/10.1080/00220612.1972.10671918

6. Learning: the treasure within; report to UNESCO of the International Commission on Education for the Twenty-first Century (highlights), The Delors Report, 1996.

7. Zhang, W.Y. (2014). Constructing China's lifelong learning "Lijiaoqiao": An international perspective. Distance Education in China, 34(6), 28-32. DOI: 10.13541/j.cnki.chinade.2014.06.004

8. The future is ours to learn, https://labour.org.uk/wpcontent/uploads/2019/11/Lifelong-Learning-Report-2019.pdf.

9. Life long learning concept https://www.ikanos.eus/wpcontent/uploads/2019/08/LifeLongLearning.Co ncept.Paper_.pdf?fbclid=IwAR1NKoG5tj2yGx4Lun0kIwyHIPu6RSXv8WVmPCXwNuCvadrBVONpkROsTMun

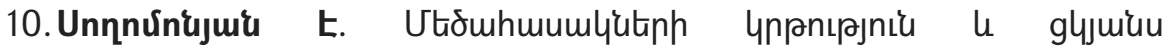

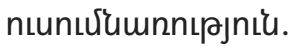

http://boon.am/andragogia-4/ 


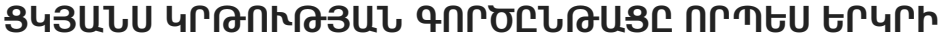

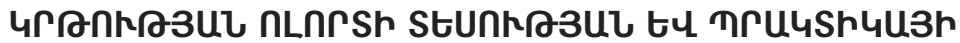 furtథחトuUu Uhহกs}

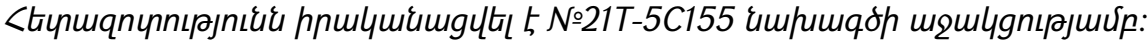

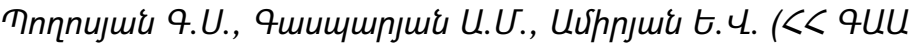

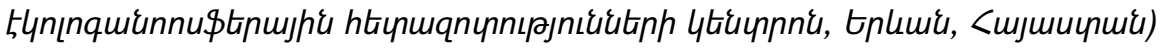

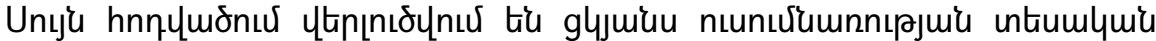

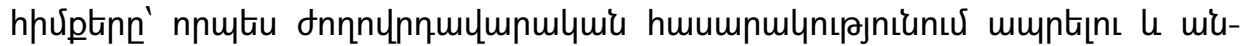

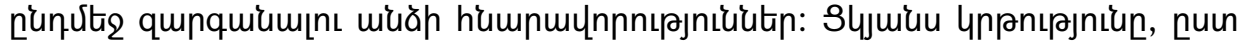

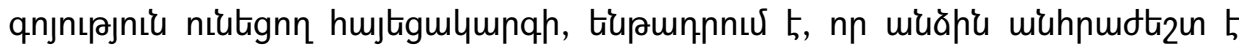

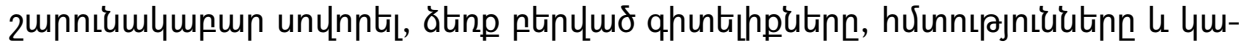

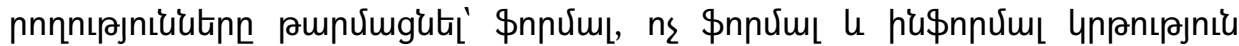

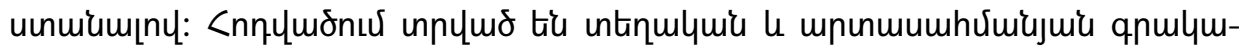

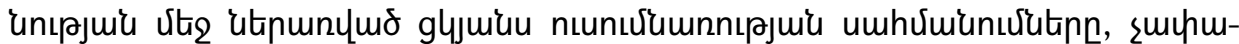

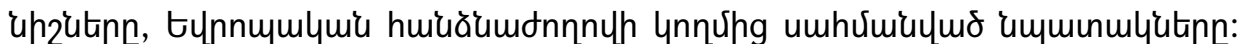

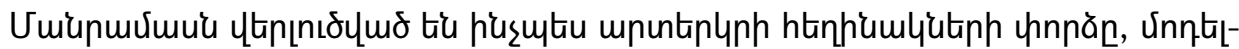

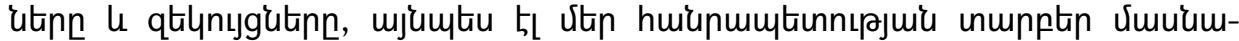

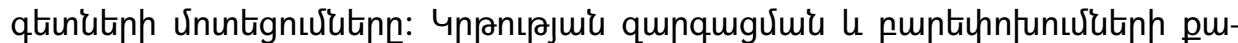

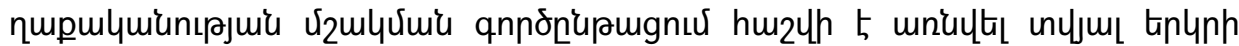

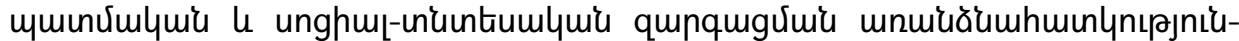

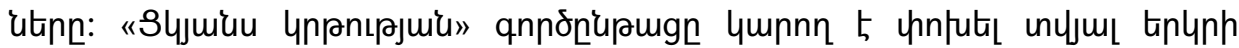

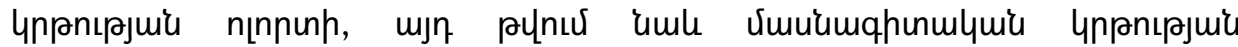
untunıpjnı'üu nı unulyunhlumu:

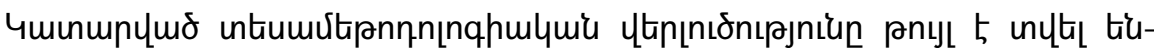

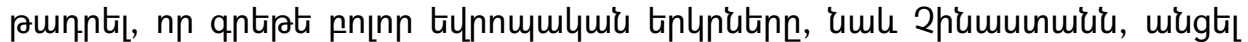

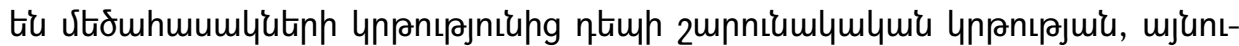

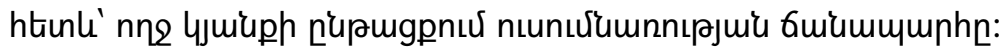

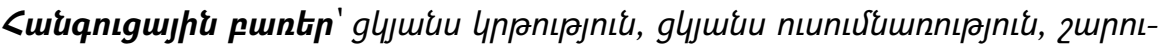

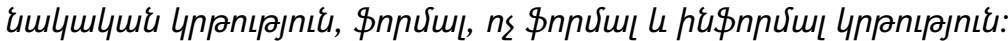

\section{ПРОЦЕСС НЕПРЕРЫВНОГО ОБРАЗОВАНИЯ КАК СРЕДСТВО РЕФОРМИРОВАНИЯ ТЕОРИИ И ПРАКТИКИ СЕКТОРА ОБРАЗОВАНИЯ СТРАНЫ}

Погосян Г. С., Гаспарян А. М., Амирян Е. В. (Начиональная академия наук Республики Армения, Ереван, Армения)

В данной статье анализируются теоретические основы непрерывного обучения как возможности для человека жить и процветать в демократичес- 
ком обществе. Непрерывное обучение, согласно существующей концепции, подразумевает, что человеку необходимо постоянно учиться, обновлять приобретенные знания, навыки и способности. На протяжении всей жизни оно должно осуществляться не только через естественное общение, жизненный опыт, но также через реализацию множества сознательных образовательных возможностей, получая формальное, неформальное, информальное образование.

В статье изложены определения, стандарты и цели обучения на протяжении всей жизни, включенные в местную и зарубежную литературу. Подробно проанализирован опыт зарубежных авторов, модели и отчеты, а также подходы различных специалистов нашей страны. Видео-методологический анализ показывает, что почти все европейские страны, а, также и Китай, перешли от образования взрослых к непрерывному образованию, а затем к обучению на протяжении всей жизни.

Ключевые слова: Образования на протяжении всей жизни, обучения на протяжении всей жизни, непрерывное образование, формальное, неформальное, информальное образование. 